\title{
Preparação e avaliação de nanocompósitos de poliestireno - hidróxido duplo lamelar HDL de ZnAl - organofuncionalizado com laurato/palmitato
}

\section{Preparation and evaluation of polystyrene (PS) - layered double hydroxide (LDH) ZnAl - organofunctionalized with laurate/palmitate nanocomposites}

\author{
Rodrigo Botan ${ }^{1}$, Núria Angelo Gonçalves ${ }^{1}$, Samara Boaventura de Moraes ${ }^{1} \mathrm{e}$ \\ Liliane Maria Ferrareso Lona ${ }^{1 *}$

\begin{abstract}
${ }^{1}$ Departamento de Engenharia de Materiais e Bioprocessos - DEMBio, Faculdade de Engenharia Química - FEQ, Universidade Estadual de Campinas - UNICAMP, Campinas, SP, Brasil
\end{abstract} \\ *liliane@feq.unicamp.br
}

\begin{abstract}
Resumo
Este trabalho descreve a síntese e caracterização de nanocompósitos de poliestireno (PS) e hidróxido duplo lamelar (HDL) formado pelos cátions de ZnAl via polimerização in situ. O HDL utilizado neste trabalho foi modificado com uma mistura de dois ânions, laurato e palmitato. Para caracterização da estrutura e morfologia dos nanocompósitos sintetizados, foram realizadas análises por difração de raios X (DRX) e microscopia eletrônica de transmissão (MET). As caracterizações térmicas, mecânicas e de flamabilidade foram realizadas via análise termogravimétrica (TGA), ensaio de flexão e ensaio de flamabilidade, respectivamente. Os resultados obtidos neste trabalho sugerem que os nanocompósitos sintetizados possuem, de uma forma geral, uma morfologia intercalada/esfoliada com melhoras em suas propriedades térmicas e mecânicas, quando comparados com o PS puro. Entretanto, não foi observado melhora na propriedade de flamabilidade.
\end{abstract}

Palavras-chave: nanocompósitos, poliestireno, hidróxido duplo lamelar, propriedade térmica, propriedade mecânica.

\section{Abstract}

This work describes the synthesis and characterization of polystyrene (PS) and layered double hydroxide (LDH) nanocomposites formed by $\mathrm{ZnAl}$ cations via in situ polymerization. The LDH used in this study was modified with a mixture of two anions: laurate and palmitate. The characterization of structure and morphology for the synthesized nanocomposites was developed by X-ray diffraction (XRD) and transmission electron microscopy (TEM). The thermal, mechanical and flammability characterizations properties were carried out via thermogravimetric analysis (TGA), bending test and flammability test, respectively. The results suggest that the nanocomposites have in general intercalated/ exfoliated morphology with improvements in thermal and mechanical properties compared to the neat PS. However no significant improvement was observed on the flammability property.

Keywords: nanocomposites, polystyrene, layered double hydroxide, thermal property, mechanical property.

\section{Introdução}

Nanocompósitos poliméricos podem ser definidos como a combinação de uma fase polimérica continua (matriz) e de outro material (reforço), sendo que, pelo menos, uma de suas dimensões apresentam-se em escala nanométrica. Atualmente a síntese e caracterização de nanocompósitos poliméricos atrai, de forma considerável, a atenção da indústria e academia. Este interesse está baseado principalmente nas melhoras em propriedades mecânicas, térmicas e físicas obtidas nestes materiais com uma pequena quantidade de reforço, geralmente com um valor máximo de $5 \%$ em massa, quando comparados a polímeros puros e compósitos tradicionais ${ }^{[1-5]}$.

Entre os diferentes tipos de nanocompósitos de matriz polimérica, os reforçados com hidróxidos duplos lamelares (HDLs) são particularmente interessantes, principalmente devido a grande versatilidade que os HDLs apresentam no seu uso em diferentes rotas de sínteses de novos nanocompósitos, bem como na melhora em propriedades térmicas, mecânicas e de flamabilidade destes materiais ${ }^{[6,7]}$. 
Os HDLs são uma classe de compostos lamelares naturais ou sintéticos que apresentam como composição química a fórmula geral $\left[\mathrm{M}^{\mathrm{II}}{ }_{1-\mathrm{x}} \mathrm{M}^{\mathrm{III}}(\mathrm{OH})_{2}\right]\left[\mathrm{A}_{\mathrm{x} / \mathrm{m}}^{\mathrm{m}-} \cdot n \mathrm{H}_{2} \mathrm{O}\right]$, onde $\mathrm{A}^{\mathrm{m}-}$ representa um ânion $\mathrm{m}$-valente, $\mathrm{n}$ representa $\mathrm{o}$ número de moléculas de água e $\mathrm{M}^{\mathrm{II}}$ e $\mathrm{M}^{\mathrm{III}}$ representam cátion di e trivalente, respectivamente ${ }^{[6,8]}$. Para melhor entendimento da estrutura dos HDLs, estes podem ser representados pela estrutura da brucita $\left(\mathrm{Mg}(\mathrm{OH})_{2}\right)$, devido à semelhança ${ }^{[8]}$, e podem ser sintetizados por meio de rotas relativamente simples com um custo relativamente baixo.

Para a efetiva síntese de nanocompósitos poliméricos com HDLs, geralmente é necessária a modificação química destes materiais, pois a maioria dos monômeros ou polímeros possuem natureza hidrofóbica, caso do estireno e PS, enquanto os HDLs são hidrofílicos, sendo incompatíveis com a fase orgânica ${ }^{[9]}$. Geralmente, esta modificação ocorre com a intercalação dos HDLs com diferentes tipos de ânions, sendo de fundamental importância salientar que HDLs com composições de cátions iguais e diferentes tipos de ânions, quando usados para síntese de nanocompósitos poliméricos, podem originar nanocompósitos com características e propriedades distintas ${ }^{[10,11]}$.

Estudos em literatura relatam a síntese de HDLs com diferentes tipos de ânions e as propriedades dos novos nanocompósitos. A maioria dos trabalhos apresentam como ânion o dodecilsulfato de sódio ${ }^{[1,2,12]}$, porém outros ânions, tais como benzoato ${ }^{[13]}$ e carboxílatos ${ }^{[10,11]}$ também são relatados.

Dos trabalhos desenvolvidos sobre nanocompósitos reforçados com HDLs, os que apresentam melhores resultados são os com HDLs modificados com carboxilatos $^{[5,10,11]}$. Entretanto, estes estudos utilizam um único ânion no espaçamento interlamelar, não sendo encontrados estudos sobre a mistura de ânions para a síntese de nanocompósitos, configurando assim, um ponto relevante a ser explorado.

A produção de nanocompósitos de matriz polimérica é uma área recente, sendo que não existe ainda uma classificação inequívoca para os diferentes tipos de materiais híbridos e respectivos métodos de síntese ${ }^{[3]}$. A síntese destes materiais, bem como suas morfologias, estão em constante desenvolvimento e, não obstante, surgem novos métodos e estruturas nunca antes relatadas.

Utilizando as rotas tradicionais e geralmente descritas para síntese dos nanocompósitos poliméricos, é possível destacar cinco vias: a mistura no fundido, esfoliação/ adsorção, síntese por solução, síntese dos nanoreforços in situ e polimerização da matriz in situ ${ }^{[3,6,14,15]}$. A polimerização in situ é particularmente interessante, uma vez que possibilita uma distribuição mais homogênea do reforço inorgânico na matriz orgânica, sendo fundamental para a síntese de materiais de alta qualidade. Em um polímero fundido, por exemplo, a distribuição do reforço não é homogênea devido à viscosidade do meio, sendo que as agregações das partículas nanométricas são difíceis de serem evitadas ${ }^{[16,17]}$.

Assim, com o objetivo de sintetizar novos nanocompósitos e estudar suas características e propriedades, este trabalho realiza a síntese in situ via polimerização em massa de nanocompósito de PS com HDL formado pelos cátions $\mathrm{ZnAl}$ modificados com uma mistura, 50\% - 50\%, dos ânions laurato e palmitato (LP). Este nanocompósito foi sintetizado com três diferentes concentrações de HDL, sendo elas $0,5,1$ e $2 \%$ em massa.

\section{Experimental}

\subsection{Materiais}

Os materiais utilizados para síntese do HDL foram ácido láurico $\left(\mathrm{C}_{12} \mathrm{H}_{24} \mathrm{O}_{2}\right)$ e ácido palmítico $\left(\mathrm{C}_{16} \mathrm{H}_{32} \mathrm{O}_{2}\right)$ (Vetec); cloreto de zinco $\left(\mathrm{ZnCl}_{2}\right)$ e cloreto de alumínio $\left(\mathrm{AlCl}_{3}\right)$ (Synth); e hidróxido de sódio $(\mathrm{NaOH})$ (Fmaia), todos em grau analítico. Para síntese dos nanocompósitos in situ foram utilizados hidróxido de sódio $(\mathrm{NaOH})$ (Fmaia), cloreto de cálcio $\left(\mathrm{CaCl}_{2}\right)$ (Ecibra), monômero (estireno) com 99\% de pureza e o iniciador Tert - Butilperóxido-2-Etilhexil Carbonato (TBEC) ambos da Sigma - Aldrich.

\subsection{Métodos}

\subsubsection{Síntese do HDL}

OHDL foi sintetizado pelo método de coprecipitação ${ }^{[18,19]}$. Para início da síntese, foram adicionados ao reator água deionizada $(\sim 600 \mathrm{~mL})$ e os ácido láurico e ácido palmítico, em uma mistura de 0,066/0,066 mol respectivamente, de um total de 0,132 mol. Após, foi adicionada uma solução básica formada por água deionizada e hidróxido de sódio, com o principal objetivo de controlar o $\mathrm{pH}$ do meio reacional em torno de 10. Após o início da adição da solução básica, foi adicionada, lentamente, ao reator (gota a gota) uma solução de sais, composta por cloreto de zinco $(0,066$ mols $)$ e cloreto de alumínio (0,033 mols) em $200 \mathrm{~mL}$ de água deionizada. Os reagentes permaneceram em agitação constante por sete horas, aproximadamente, para término da adição dos reagentes. Todo esse processo de síntese também foi acompanhado por um controle de $\mathrm{pH}$, temperatura $\left(35^{\circ} \mathrm{C}\right)$ e atmosfera de nitrogênio, com o objetivo de manter o sistema inerte.

Terminada a adição dos reagentes, a suspensão formada foi mantida em repouso no reator por 12 horas, para estabilização dos cristais. Após este período, o HDL foi precipitado e lavado com água deionizada em uma centrífuga da marca Sigma, modelo 3 - 16P. Esse processo de lavagem foi repetido por cinco vezes. Após a lavagem, o HDL foi seco em uma estufa a vácuo da marca Tecnal, modelo TE 395 , com temperatura em $50^{\circ} \mathrm{C}$

\subsubsection{Síntese do PS puro e nanocompósitos}

Para síntese dos nanocompósitos foi utilizada a polimerização em massa via radical livre. Neste trabalho foram sintetizados o PS puro e os nanocompósitos. Estes nanocompósitos foram produzidos com diferentes percentuais de carga inorgânica (HDL) $(0,5 \% ; 1 \%$ e $2 \%$ em massa). A primeira tarefa realizada antes do procedimento experimental de polimerização foi a purificação do monômero ${ }^{[20]}$. O monômero foi lavado por três vezes com uma solução de $\mathrm{NaOH}$ e três vezes com água deionizada. Depois de lavado, este monômero foi seco com $\mathrm{CaCl}_{2}$.

Para a polimerização do PS puro foram utilizados monômero e iniciador, e para os nanocompósitos foram 
utilizados o mesmo monômero e iniciador com o acréscimo do HDL. As reações de polimerização em massa para a produção do polímero puro ${ }^{[20]} \mathrm{e}$ dos nanocompósitos foram realizadas em ampolas e ocorreram em um regime de batelada a uma temperatura de $108^{\circ} \mathrm{C}$ durante um período de três horas e meia em banho com circulação de fluido. Nestas reações o iniciador TBEC foi utilizado na concentração de $0,036 \mathrm{~mol} / \mathrm{L}$.

\subsection{Caracterização}

Para caracterização dos materiais obtidos neste trabalho foram utilizadas as técnicas de DRX, realizada através de um equipamento da marca Shimadzu, modelo XRD 7000. Este equipamento utilizou um cátodo de cobre com comprimento de onda $1,5406 \AA$, potência da fonte de $40 \mathrm{kV}$ e corrente de $30 \mathrm{~mA}$. A velocidade de varredura utilizada foi de $2^{\circ}$ por minuto e a faixa varrida foi de $(2 \theta) 1,5^{\circ}$ até $50^{\circ}$.

$\mathrm{Na}$ análise do MET foi utilizado o equipamento modelo JEM 3010, da marca Jeol com uma aceleração de voltagem de $300 \mathrm{kV}$. As amostras utilizadas nesta análise apresentavam uma espessura de $120 \mathrm{~nm}$ e foram cortadas, com faca de vidro, em um ultramicrótomo e em temperatura ambiente.

As medidas de TGA foram realizadas no equipamento da marca TA Instruments, modelo TGA 2050. Neste ensaio foi utilizada atmosfera oxidante com fluxo de $100 \mathrm{~mL} / \mathrm{min}$ e taxa de aquecimento de $10^{\circ} \mathrm{C} / \mathrm{min}$. Utilizou-se uma faixa de aquecimento de $25^{\circ} \mathrm{C}$ (temperatura ambiente) até $700^{\circ} \mathrm{C}$.

Para realização dos testes de flamabilidade e ensaio mecânico, foram confeccionados corpos de prova com dimensões de $64 \times 12,7 \times 3,15 \mathrm{~mm}$ através da injeção em uma mini-injetora da marca Thermo Fisher Scientific, modelo HaakeMiniJet II.

O teste de flamabilidade realizado foi baseado na norma ASTM D635 (UL 94 HB), com modificação no corpo de prova devido à escassez de material, obtido por síntese em ampola. Neste teste foram feitos três ensaios para cada tipo de material sintetizado e em posição horizontal. As taxas de queima dos materiais foram medidas e utilizadas como comparação entre o polímero puro e nanocompósitos.
O ensaio mecânico realizado neste trabalho foi o de flexão em três pontos, baseado na norma ASTM D790. Devido à escassez de material obtido mediante síntese em ampolas, foram realizados três ensaios para cada material sintetizado. Estes ensaios mecânicos foram realizados em uma máquina universal de ensaios da marca MTS, modelo MTS 810. A taxa de deformação utilizada neste ensaio foi de $0,01 \mathrm{~mm} / \mathrm{mm} / \mathrm{min}$.

\section{Resultados e Discussão}

\subsection{Estrutura e morfologia dos nanocompósitos de PS/ $Z n A l-L P$}

As primeiras técnicas de caracterizações utilizadas nos nanocompósitos sintetizados in situ neste trabalho foram a DRX e o MET, com o principal objetivo de confirmar a síntese dos nanocompósitos e definir as suas morfologias.

A Figura 1 apresenta os difratogramas de raios X para o ZnAl - LP, PS puro e nanocompósitos de PS/ZnAl - LP.

O difratograma para o ZnAl - LP como pode ser observado, apresenta séries de picos basais característicos deste tipo de material ${ }^{[8,14,21-23]}$, o que sugere a síntese deste HDL. Entretanto, diferentemente dos difratogramas observados para HDLs sintetizados com apenas um ânion, o difratograma do ZnAl - LP apresenta duas séries de picos basais, sugerindo que existe uma série referente a parte do material intercalado preferencialmente com o ânion palmitato e outra série intercalada preferencialmente com o ânion laurato. Quando as séries basais encontradas neste trabalho são comparadas com as séries dos ânions intercalados sem mistura nos HDLs, existe similaridade ${ }^{[21-23]}$. Através das séries de picos basais obtidos no difratograma do $\mathrm{ZnAl}$ - LP e da lei de Bragg, é possível calcular o espaçamento basal, característica de relevante importância deste material. Devido às duas séries de picos encontrados, foram obtidos dois valores de espaçamento basais, sendo elas de 38,42 À e 33,92 A.

Assim, comparando os valores de espaçamentos basais obtidos, os ânions utilizados e os resultados dos valores dos espaçamentos basais relatados em literatura, é possível

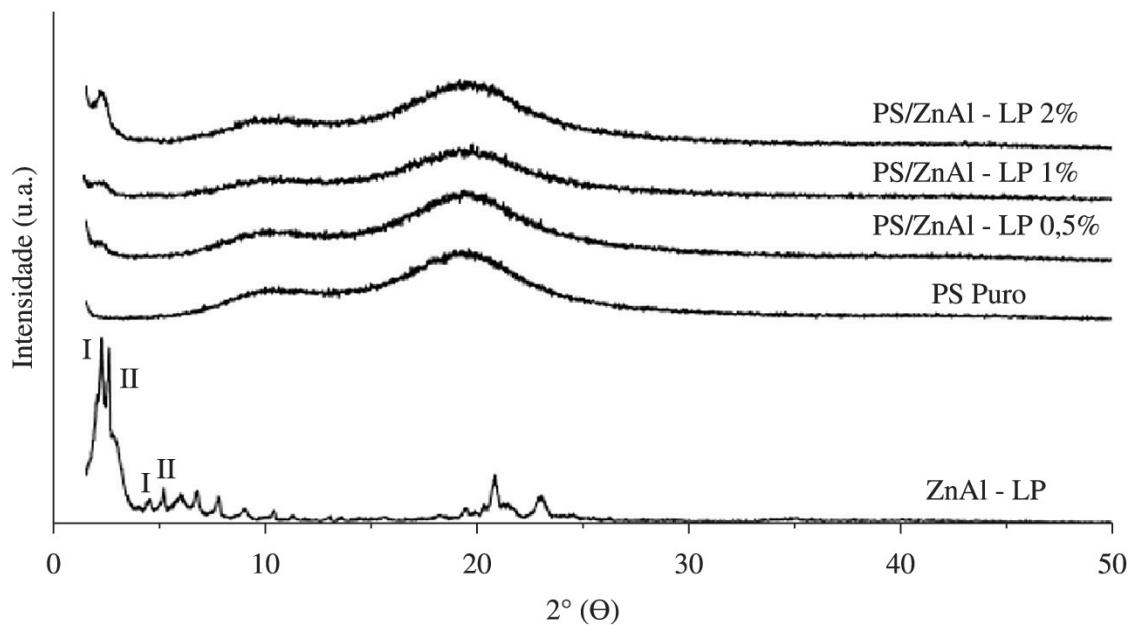

Figura 1. Difratogramas de raios $\mathrm{X}$ obtidos para os materiais sintetizados; ZnAl - LP, PS puro e nanocompósitos. 
sugerir que a primeira série de pico com espaçamento basal de $38,42 \AA$ é referente a parte do material intercalada com o ânion palmitato e a segunda série é referente a parte do material intercalada com o ânion laurato ${ }^{[8,14,21-23]}$. Na Figura 1 as primeiras séries de picos (003) e (006), são identificadas com I para a parte intercalada com palmitato e II intercalada com laurato.

Quando são comparados os resultados obtidos com o valor de espaçamento basal do ZnAl sem modificação (com ânion cloreto), 7,74 $\AA^{[8,19,22]}$, pode-se observar mais um indício de que a modificação do HDL com os ânions selecionados ocorreu de forma efetiva. Quando o HDL, neste caso o ZnAL, é modificado com os ânions selecionados, estes ânions, devido ao seu maior tamanho, expandem o seu espaçamento basal. Assim, quando comparado o valor do $\mathrm{ZnAl}$ modificado com o do $\mathrm{ZnAl} \mathrm{sem}$ modificação, irá existir uma diferença referente ao ânion intercalante ${ }^{[21-23]}$, como demonstram os resultados obtidos.

Para a efetiva síntese dos nanocompósitos, a expansão do espaçamento basal dos HDLs é de fundamental importância, pois através deste aumento as forças que mantêm as lamelas do HDL unidas diminuem, permitindo a adsorção de compostos orgânicos neste domínio. Assim, torna-se possível que, na síntese in situ, o monômero adsorva entre as lamelas do HDL e quando polimerizado possa ocorrer a delaminação da estrutura do HDL e consequentemente a produção do nanocompósito ${ }^{[1,2,9]}$.

Após a caracterização do $\mathrm{ZnAl}$ - LP através da DRX, este mesmo ensaio foi realizado no PS puro e nanocompósitos.

O PS puro, como pode ser observado, não apresenta nenhum pico característico na DRX. Existem apenas dois pequenos halos em torno de 10 e $20^{\circ}(2 q)$, característicos da sua natureza amorfa ${ }^{[1,9]}$.

Analisando os difratogramas dos nanocompósitos, é possível concluir que estes materiais apresentam de uma forma geral, seus difratogramas semelhantes aos do PS puro, com dois pequenos halos em torno de 10 e $20^{\circ}$ (2q). Entretanto, uma diferenciação pode ser observada no início dos difratogramas.

Em todos os nanocompósitos sintetizados pode-se observar, no início dos difratogramas (em valores de baixos ângulos), a formação de um leve pico. A existência deste pico demostra que existe na estrutura dos nanocompósitos algum tipo de organização do $\mathrm{ZnAl}$ - LP. Com a presença desta organização do reforço na matriz polimérica, é possível indicar que este $\mathrm{ZnAl}$ - LP não apresenta uma ótima distribuição global na matriz polimérica, sugerindo a formação de pequenos aglomerados (tactóides) deste reforço em todos os nanocompósitos sintetizados.

Para complementar as informações obtidas nas análises de DRX e definir quais estruturas e morfologias os nanocompósitos sintetizados possuem, estes foram caracterizados pelo MET.

Geralmente, no MET, são realizadas imagens em baixa resolução, para uma visualização de como o reforço está distribuído na matriz polimérica, e em alta resolução, para identificar a morfologia dos nanocompósitos ${ }^{[1,6,9,10]}$.

A Figura 2 apresenta as imagens do MET para o nanocompósito de PS/ZnAl - LP 2\%. Como pode ser observado, a imagem em baixa resolução (Figura 2a) deste nanocompósito, reforça o resultado obtido na DRX (pico em baixo ângulo), em que pode ser visualizado uma pobre distribuição global do reforço na matriz polimérica. Nesta imagem são identificadas a existência de aglomerados, tactóides (indicados pelas setas brancas), caracterizando uma concentração do reforço em alguns pontos da matriz polimérica.

Quando é analisada a imagem do MET em alta resolução (Figura 2b), é possível identificar que, apesar da pobre distribuição do reforço na matriz polimérica,

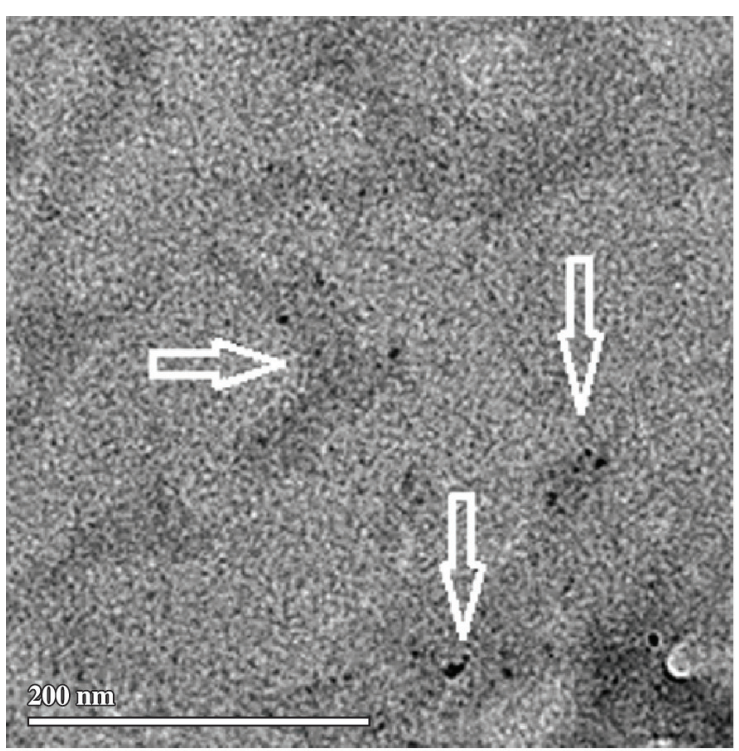

(a)

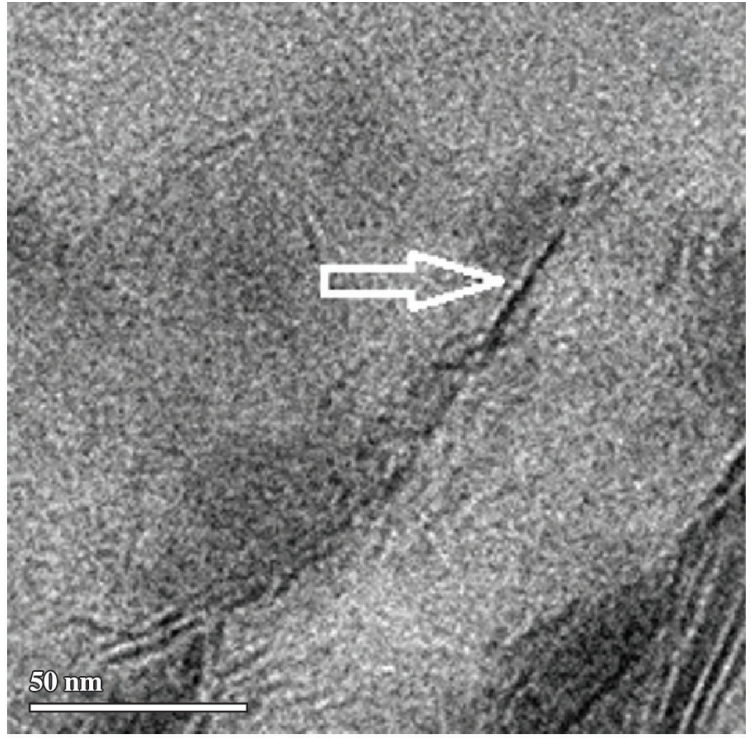

(b)

Figura 2. Imagens do MET para o nanocompósito de PS/ZnAl - LP 2\% em (a) baixa resolução e em (b) alta resolução. 
são identificadas regiões em grande maioria com lamelas intercaladas e regiões com lamelas esfoliadas (seta branca).

Assim, através da MET é possível sugerir que este nanocompósito apresenta uma pobre distribuição global do reforço na matriz polimérica, entretanto mesmo com essa pobre distribuição global, o material apresenta em sua matriz polimérica lamelas intercaladas, em maior quantidade, e lamelas esfoliadas em pequena quantidade, caracterizando uma morfologia intercalada com algumas lamelas esfoliadas, intercalada/esfoliada.

\subsection{Propriedade térmica dos nanocompósitos de PS/ $Z n A l-L P$}

Uma das propriedades de maior importância para o desenvolvimento e aplicação dos materiais poliméricos são suas propriedades térmicas. Neste trabalho, para a caracterização térmica do PS puro e nanocompósitos, foi realizada a TGA. Os resultados obtidos da TGA são apresentados na Figura 3.

O PS puro apresentou neste ensaio uma característica muito marcante e conhecida. Este material começou a se degradar, ou seja, perder massa, a uma temperatura em torno de $300^{\circ} \mathrm{C}$ e a partir deste ponto, as cadeias poliméricas do PS são degradadas até valores de temperatura em torno de $385^{\circ} \mathrm{C}$, temperatura na qual quase a totalidade deste material foi consumido.

As curvas de TGA de todos os nanocompósitos, como podem ser observadas, seguem o mesmo padrão do PS puro, em que estes materiais começam a degradar a uma temperatura acima de $\sim 310^{\circ} \mathrm{C}$ e a partir deste ponto, os nanocompósitos são degradados até valores de temperatura em torno de $390^{\circ} \mathrm{C}$, temperatura na qual quase a totalidade destes materiais foram consumidos.

É importante ressaltar que, mesmo seguindo o padrão do PS puro, todas as curvas dos nanocompósitos sintetizados apresentaram uma degradação mais lenta quando comparados com o PS puro, demonstrando que estes novos nanocompósitos apresentaram uma melhora em suas estabilidades térmicas.

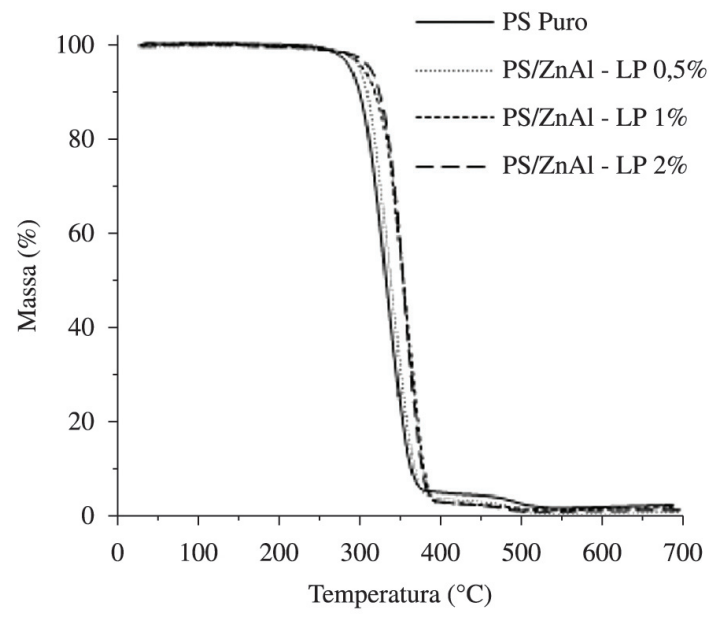

Figura 3. Curvas da TGA do PS puro e nanocompositos de PS/ $\mathrm{ZnAl}-\mathrm{LP}$.
Esta melhora na estabilidade térmica deve-se, primordialmente, à interação que ocorre entre o polímero e o HDL. Nestes nanocompósitos existem reforços em escala nanométrica, criando uma grande área superficial de contato entre HDL e PS. Com esta grande área de contato, a mobilidade das cadeias poliméricas torna-se mais difícil, bem como existe uma maior resistência à difusão de oxigênio e compostos voláteis, acarretando, de forma geral, em uma degradação mais lenta apresentada pelos nanocompósitos ${ }^{[1-5]}$.

Para um estudo mais completo do comportamento térmico do PS puro e nanocompósitos, valores específicos obtidos nestas curvas foram analisados.

O primeiro ponto de análise foi a temperatura de início da degradação $\left(\mathrm{T}_{\text {ini }}\right)$, definida como a temperatura onde começa a degradação do material polimérico ou nanocompósito e obtida na curva de TGA, quando $10 \%$ do material foi degradado. O segundo ponto foi a temperatura da metade da degradação $\left(\mathrm{T}_{\mathrm{me}}\right)$, definida como a temperatura onde ocorre metade da degradação do material polimérico ou nanocompósito e obtida na curva de TGA, quando $50 \%$ do material foi degradado. O terceiro ponto foi a temperatura final da degradação do material $\left(\mathrm{T}_{\mathrm{fin}}\right)$, sendo definida como a temperatura onde quase todo o material foi degradado e obtida na curva de TGA, quando $90 \%$ do material foi degradado.

Os valores obtidos para estas temperaturas são apresentados na Tabela 1.

Assim, através dos resultados obtidos, é possível constatar a melhora obtida na estabilidade térmica destes novos nanocompósitos, quando comparados com o PS puro, em todas as composições utilizadas.

É importante salientar que os resultados de melhora obtidos na TGA no presente trabalho são um avanço no estudo de nanocompósitos de PS com HDL, pois em estudos desenvolvidos em nosso grupo de pesquisa utilizando outro ânion no HDL, dodecil sulfato de sódio ${ }^{[1,9]}$, foram obtidas melhoras de $10^{\circ} \mathrm{C}$ na temperatura de $\mathrm{T}_{\text {me }}$ com o uso de $1 \%$ de HDL no nanocompósito. Neste trabalho com $1 \%$ de HDL na temperatura de $\mathrm{T}_{\text {me }}$ foi obtida uma melhora de $22^{\circ} \mathrm{C}$. Os resultados obtidos neste trabalho também apresentam melhoras quando comparados com outros trabalhos existentes em literatura que sintetizam nanocompósitos de PS e HDL ${ }^{[10,24,25]}$.

\subsection{Flamabilidade dos nanocompósitos de PS/ZnAl - LP}

O teste de flamabilidade realizado neste trabalho consiste em submeter os materiais sintetizados a condições extremas, sendo colocados em contato direto com a chama e, a partir de um tempo de ignição, é medido o tempo de

Tabela 1. Temperaturas obtidas na TGA para o PS puro e nanocompósitos sintetizados.

\begin{tabular}{cccc}
\hline \multirow{2}{*}{ Materiais } & \multicolumn{3}{c}{ Temperaturas $\left({ }^{\circ} \mathbf{C}\right)$} \\
\cline { 2 - 4 } & $\mathbf{T}_{\text {ini }}$ & $\mathbf{T}_{\text {me }}$ & $\mathbf{T}_{\text {fin }}$ \\
\hline PS Puro & 299 & 332 & 363 \\
PS/ZnAl - LP 0,5\% & 308 & 338 & 368 \\
PS/ZnAl - LP 1\% & 319 & 354 & 379 \\
PS/ZnAl - LP 2\% & 324 & 354 & 377 \\
\hline
\end{tabular}


combustão para cada amostra. Este ensaio foi realizado com o objetivo de comparar a flamabilidade do PS puro e nanocompósitos sintetizados.

Os resultados obtidos no teste de flamabilidade são apresentados na Figura 4. No gráfico é possível identificar que a diferença encontrada para o PS puro e nanocompósitos de PS/ZnAl - LP foram muito pequenas, onde a maioria dos valores médios estão dentro ou muito próximos à variação dos resultados.

Assim, é possível concluir que não houve diferença significativa na propriedade de flamabilidade quando comparamos o PS puro e os nanocompósitos sintetizados.

Outros fatores relevantes que podem ser identificados neste ensaio são o fenômeno de gotejamento e a emissão de fuligem (fumaça). Estes fatores são muito importantes em situações de risco, pois através do gotejamento o fogo é espalhado para outros materiais, bem como a emissão da fuligem (fumaça) em grandes quantidades diminui a visibilidade, além de ser considerada tóxica, podendo levar à parada respiratória.

Assim, quanto menor o gotejamento e a emissão de fuligem mais seguro é o material em situações de perigo. As análises de gotejamento e emissão de fuligem neste ensaio foram identificadas por observação direta destes fenômenos ${ }^{[1,4,17]}$.

O PS puro tem como característica apresentar um gotejamento moderado e a emissão de um grande volume de fuligem. Quando comparamos os resultados do PS puro com os nanocompósitos sintetizados não foi identificada nenhuma diferença. Estes nanocompósitos em todas as composições utilizadas apresentaram, assim como o PS puro, um gotejamento moderado e a emissão de um volume de fuligem considerável durante a combustão.

\subsection{Propriedade mecânica dos nanocompósitos de PS/ $\mathrm{ZnAl}$ - LP}

A determinação das propriedades mecânicas para polímeros e nanocompósitos poliméricos é de fundamental importância, pois a grande maioria das aplicações destes materiais, de alguma forma, depende das suas propriedades mecânicas. Apesar da grande importância das propriedades

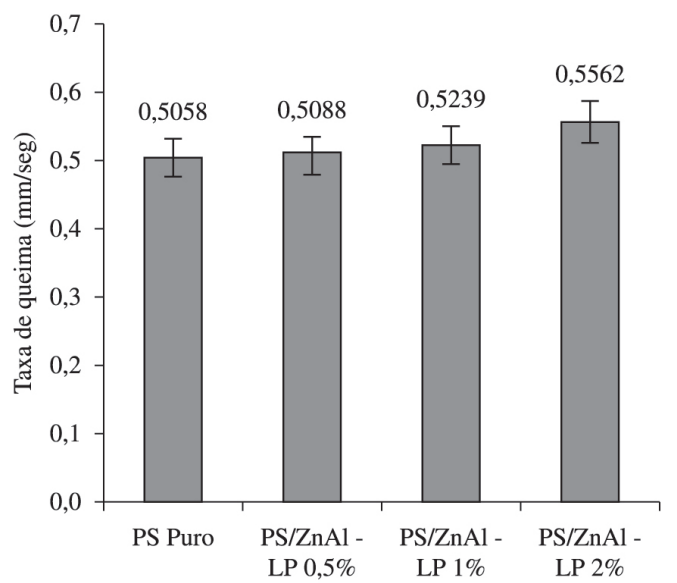

Figura 4. Resultado da taxa de queima para o ensaio de flamabilidade do PS puro e nanocompósitos mecânicas, estudos sobre estas propriedades para nanocompósitos reforçados com HDLs ainda são escassos.

A propriedade mecânica dos materiais sintetizados neste trabalho foi obtida através do ensaio de flexão. O ensaio de flexão foi escolhido devido à fragilidade do PS puro e seus nanocompósitos, o que impossibilita resultados confiáveis no ensaio de tração. A Figura 5 apresenta as curvas dos ensaios de flexão para o PS puro e nanocompósitos.

É importante salientar que, nas curvas dos ensaios de flexão para o PS puro e nanocompósitos, em todos os casos, ocorreu o rompimento, ou quebra, do material no valor máximo de tensão de flexão. A continuação da linha após o rompimento foi mantida para melhor identificação dos materiais ${ }^{[26]}$.

A Tabela 2 mostra os valores encontrados para a tensão de flexão, deformação na quebra e módulo de flexão do PS puro e nanocompósitos de PS/ZnAl - LP. Os resultados apresentados são a média dos resultados obtidos (três ensaios) e os desvios percentuais $( \pm \%)$ de cada material sintetizado, sendo que o \pm desvio percentual foi calculado pela seguinte formula: |(valor medido-média dos valores)/ média dos valores x $\left.100 \%\right|^{[26]}$.

Estudando os resultados obtidos, é possível observar que todos os nanocompósitos sintetizados em todas as composições, apresentaram aumento das propriedades mecânicas quando comparados ao PS puro.

Para os valores obtidos de tensão de flexão e deformação na quebra, é possível observar um padrão em todos os nanocompósitos: com o aumento da quantidade de HDL, ocorre um aumento nos valores destas propriedades.

Comparando o resultado obtido da tensão de flexão para o PS puro com o do nanocompósito com $0,5 \%$ de reforço, é possível observar que este material apresenta, em média, um valor $27 \%$ maior. Este valor, para o nanocompósito com $1 \%$ aumenta em $46 \%$ e para o nanocompósito com $2 \%$ ocorreu um aumento médio de $62 \%$.

Estes resultados demonstram que os novos nanocompósitos sintetizados apresentaram ganhos consideráveis nas propriedades mecânicas medidas, quando comparadas com o PS puro. Este ganho pode ser explicado pela grande área superficial de contato entre HDL e PS.

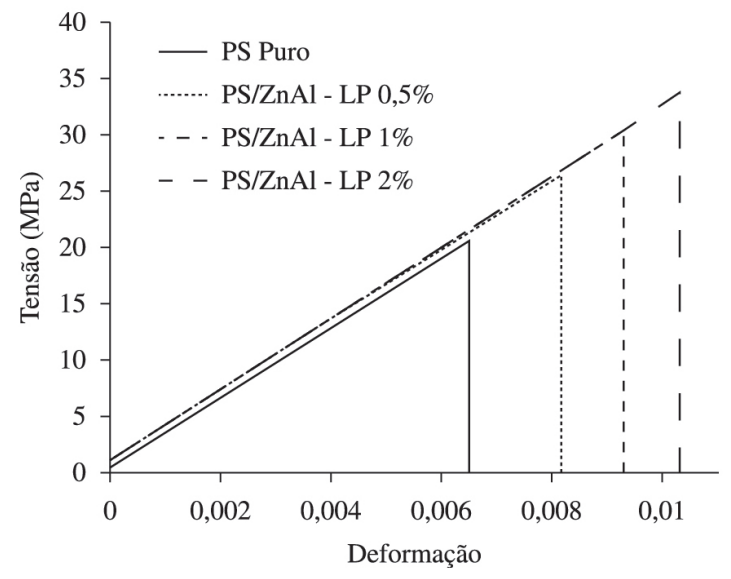

Figura 5. Curvas dos ensaios de flexão para o PS puro e nanocompósitos de PS/ZnAl - LP. 
Tabela 2. Valores da tensão de flexão, da deformação na quebra e do modulo de flexão com seus desvios percentuais para o ensaio de flexão realizado no PS puro e nanocompósitos.

\begin{tabular}{cccc}
\hline Materiais & Tensão de Flexão (Mpa) (\%) & Deformação (\%) & Módulo de Flexão (Mpa) (\%) \\
\hline PS puro & $20,62 \pm 3,59$ & $0,65 \pm 1,51$ & $3.186 \pm 0,81$ \\
PS/ZnAl - LP 0,5\% & $26,37 \pm 7,83$ & $0,82 \pm 8,14$ & $3.231 \pm 0,56$ \\
PS/ZnAl - LP 1\% & $30,13 \pm 8,45$ & $0,93 \pm 8,73$ & $3.241 \pm 1,25$ \\
PS/ZnAl - LP 2\% & $33,49 \pm 2,84$ & $1,05 \pm 1,94$ & $3.220 \pm 0,84$ \\
\hline
\end{tabular}

Com maior área de contato, a mobilidade das cadeias poliméricas torna-se mais difícil, uma vez que o HDL age como barreira ao movimento das cadeias, melhorando assim as propriedades mecânicas dos nanocompósitos ${ }^{[26,27]}$.

Os resultados do módulo de flexão dos nanocompósitos sintetizados, assim como a tensão de flexão e deformação na quebra, apresentaram melhora quando comparada com o PS puro.

Quando são comparados os resultados de propriedades mecânicas obtidas neste trabalho com os poucos resultados encontrados em literatura pode ser observado um avanço, pois nos trabalhos encontrados em literatura para síntese e caracterização de nanocompósitos de PS e HDL não há melhora nas propriedades mecânicas, sendo que há piora nestas propriedades em todas as composições sintetizadas $^{[10,25]}$.

\section{Conclusões}

Neste trabalho foram sintetizados, com sucesso, novos nanocompósitos de PS/ZnAl - LP. Estes nanocompósitos foram caracterizados pelas técnicas de DRX e MET, as quais identificaram que o $\mathrm{ZnAl}$ - LP apresentou uma pobre distribuição na matriz polimérica, bem como a morfologia intercalada/esfoliada dos nanocompósitos. Todos os nanocompósitos apresentaram melhor estabilidade térmica quando comparados com o PS puro. O ganho na temperatura da metade da degradação $\left(\mathrm{T}_{\mathrm{me}}\right)$ para o nanocompósito com maior quantidade de HDL, $2 \%$, foi de $22^{\circ} \mathrm{C}$. A flamabilidade dos nanocompósitos também foi medida e comparada com a do PS puro, sendo que os resultados obtidos não apresentaram diferenças significativas, assim não existe diferença entre PS puro e nanocompósitos sintetizados em relação a esta propriedade. Entretanto, para as propriedades mecânicas, ocorreram ganhos consideráveis. A tensão de flexão do nanocompósito com $2 \%$ de HDL apresentou um ganho em média de $62 \%$ quando comparado com o do PS puro.

\section{Agradecimentos}

Os autores agradecem a CAPES pelo auxílio financeiro e ao Laboratório Nacional de Luz Sincrotron (LNLS) pelo apoio ao projeto, disponibilizando a sua infraestrutura laboratorial para as análises do MET.

\section{Referências}

1. Botan, R., Nogueira, T. R., Wypych, F., \& Lona, L. M. F. (2012). In situ synthesis, morphology, and thermal properties of polystyrene-MgAl layered double hydroxide nanocomposites. Polymer Engineering and Science, 52(8), 1754-1760. http://dx.doi.org/10.1002/pen.23122.
2. Peng, H., Tjiu, W. C., Shen, L., Huang, S., He, C., \& Liu, T. (2009). Preparation and mechanical properties of exfoliated CoAl layered double hydroxide (LDH)/polyamide 6 nanocomposites by in situ polymerization. Composites Science and Technology, 69(7-8), 991-996. http://dx.doi. org/10.1016/j.compscitech.2009.01.005.

3. Esteves, A. C. C., Barros-Timmons, A. B., \& Trindade, T. (2004). Nanocompósitos de matriz polimérica: estratégias de síntese de materiais híbridos. Quimica Nova, 27(5), 798-806. http://dx.doi.org/10.1590/S0100-40422004000500020.

4. Matusinovic, Z., \& Wilkie, C. A. (2012). Fire retardancy and morphology of layered double hydroxide nanocomposites: a review. Journal of Materials Chemistry, 22(36), 18701. http:// dx.doi.org/10.1039/c2jm33179a.

5. Nogueira, T., Botan, R., Macedo Neto, J. C., Wypych, F., \& Lona, L. (2013). Effect of Layered Double Hydroxides on the Mechanical, Thermal, and Fire Properties of Poly(methyl methacrylate) Nanocomposites. Advances in Polymer Technology, 32(S1), E660-E674. http://dx.doi.org/10.1002/ adv.21309.

6. Wypych, F., \& Satyanarayana, K. G. (2005). Functionalization of single layers and nanofibers: a new strategy to produce polymer nanocomposites with optimized properties. Journal of Colloid and Interface Science, 285(2), 532-543. http://dx.doi. org/10.1016/j.jcis.2004.12.028. PMid:15837469

7. Paul, D. R., \& Robeson, L. M. (2008). Polymer nanotechnology: Nanocomposites. Polymer, 49(15), 3187-3204. http://dx.doi. org/10.1016/j.polymer.2008.04.017.

8. Rives, V. (2006). Layered double hydroxide: Present and future. New York: Nova Science Publishers.

9. Botan, R., Nogueira, T. R., Lona, L. M. F., \& Wypych, F. (2011). Synthesis and characterization of Exfoliated Polystyrene: Layered Double Hydroxide Nanocomposites via in situ polymerization. Polímeros Ciência e Tecnologia, 21(1), 34-38. http://dx.doi.org/10.1590/S0104-14282011005000017.

10. Nyambo, C., Songtipya, P., Manias, E., Jimenez-Gasco, M. M., \& Wilkie, C. A. (2008). Effect of MgAl-layered double hydroxide exchanged with linear alkyl carboxylates on fireretardancy of PMMA and PS. Journal of Materials Chemistry, 18(40), 4827. http://dx.doi.org/10.1039/b806531d.

11. Nogueira, T., Botan, R., Wypych, F., \& Lona, L. (2012). Synthesis and characterization of LDHs/PMMA nanocomposites: Effect of two different intercalated anions on the mechanical and thermal properties. Journal of Applied Polymer Science, 124(3), 1764-1770. http://dx.doi. org/10.1002/app.35213.

12. Tai, Q., Chen, L., Song, L., Hu, Y., \& Yuen, R. K. K. (2011). Effects of a phosphorus compound on the morphology, thermal properties, and flammability of polystyrene/MgAl-layered double hydroxide nanocomposites. Journal of Polymer Composites, 32(2), 168-176. http://dx.doi.org/10.1002/ pc. 21022 .

13. Matusinovic, Z., Feng, J., \& Wilkie, C. A. (2013). The role of dispersion of LDH in fire retardancy: The effect of different divalent metals in benzoic acid modified LDH on dispersion and fire retardant properties of polystyreneand poly(methyl-methacrylate)-LDH-B nanocomposites. 
Polymer Degradation \& Stability, 98(8), 1515-1525. http:/ dx.doi.org/10.1016/j.polymdegradstab.2013.04.007.

14. Khan, A. I., \& O'Hare, D. (2002). Intercalation chemistry of layered double hydroxides: recent developments and applications. Journal of Materials Chemistry, 12(11), 31913198. http://dx.doi.org/10.1039/b204076j.

15. Acharya, H., Srivastava, S. K., \& Bhowmick, A. K. (2007). Synthesis of partially exfoliated EPDM/LDH nanocomposites by solution intercalation: Structural characterization and properties. Composites Science and Technology, 67(13), 28072816. http://dx.doi.org/10.1016/j.compscitech.2007.01.030.

16. Demir, M. M., Memesa, M., Castignolles, P., \& Wegner, G. (2006). PMMA/Zinc Oxide Nanocomposites Prepared by In-Situ Bulk Polymerization. Macromolecular Rapid Communications, 27(10), 763-770. http://dx.doi.org/10.1002/ marc. 200500870 .

17. Moraes, S. B., Botan, R., \& Lona, L. M. F. (2014). Síntese e caracterização de nanocompósitos de poliestireno/hidroxissal lamelar. Quimica Nova, 37(1), 18. http://dx.doi.org/10.1590/ S0100-40422014000100004.

18. Iyi, N., Tamura, K., \& Yamada, H. (2009). One-pot synthesis of organophilic layered double hydroxides (LDHs) containing aliphatic carboxylates: extended "homogeneous precipitation" method. Journal of Colloid and Interface Science, 340(1), 67-73. http://dx.doi.org/10.1016/j.jcis.2009.08.026. PMid:19762037

19. Nhlapo, N., Motumi, T., Landman, E., Verryn, S. M. C., \& Focke, W. W. (2008). Surfactant-assisted fatty acid intercalation of layered double hydroxides. Journal of Materials Science, 43(3), 1033-1043. http://dx.doi. org/10.1007/s10853-007-2251-0.

20. Nogueira, T. R., Lona, L. M. F., Mcmanus, N. T., VivaldoLima, E., \& Penlidis, A. (2010). Nitroxide-mediated radical copolymerization of styrene and divinylbenzene: increased polymerization rate by using TBEC as initiator. Journal of Materials Science, 45(7), 1878-1884. http://dx.doi. org/10.1007/s10853-009-4172-6.

21. Xu, Z. P., Braterman, P. S., Yu, K., Xu, H., Wang, Y., \& Brinker, C. J. (2004). Unusual Hydrocarbon Chain Packing Mode and Modification of Crystallite Growth Habit in the Self-Assembled Nanocomposites Zinc-Aluminum-Hydroxide
Oleate and Elaidate ( cis - and trans - $[\mathrm{Zn} 2 \mathrm{Al}(\mathrm{OH}) 6(\mathrm{CH}$ $3(\mathrm{CH} 2$ ) $7 \mathrm{CHCH}(\mathrm{CH} 2) 7 \mathrm{COO}-)]$ and Magnesium Analogues. Chemistry of Materials, 16(14), 2750-2756. http:// dx.doi.org/10.1021/cm0497529.

22. Xu, Z. P., \& Braterman, P. S. (2010). Synthesis, structure and morphology of organic layered double hydroxide (LDH) hybrids: Comparison between aliphatic anions and their oxygenated analogs. Applied Clay Science, 48(1-2), 235-242. http://dx.doi.org/10.1016/j.clay.2009.11.009.

23. Kuehn, T., \& Poellmann, H. (2010). Synthesis and characterization of $\mathrm{Zn}$-Al layered double hydroxides intercalated with 1- to 19-carbon carboxylic acid anions. Clays and Clay Minerals, 58(5), 596-605. http://dx.doi.org/10.1346/ CCMN.2010.0580502.

24. Manzi-Nshuti, C., Chen, D., Su, S., \& Wilkie, C. A. (2009). Structure-property relationships of new polystyrene nanocomposites prepared from initiator-containing layered double hydroxides of zinc aluminum and magnesium aluminum. Polymer Degradation \& Stability, 94(8), 1290-1297. http://dx.doi.org/10.1016/j.polymdegradstab.2009.03.021.

25. Nyambo, C., Wang, D., \& Wilkie, C. A. (2009). Will layered double hydroxides give nanocomposites with polar or nonpolar polymers? Polymers for Advanced Technologies, 20(3), 332-340. http://dx.doi.org/10.1002/pat.1272.

26. Ayewah, D. O. O., Davis, D. C., Krishnamoorti, R., Lagoudas, D. C., Sue, H. J., \& Willson, M. A. (2010). A surfactant dispersed SWCNT-polystyrene composite characterized for electrical and mechanical properties. Composites. Part A, Applied Science and Manufacturing, 41(7), 842-849. http:// dx.doi.org/10.1016/j.compositesa.2010.02.015.

27. Nogueira, T., Botan, R., Wypych, F., \& Lona, L. (2011). Study of thermal and mechanical properties of PMMA/LDHs nanocomposites obtained by in situ bulk polymerization. Composites. Part A, Applied Science and Manufacturing, 42(8), 1025-1030. http://dx.doi.org/10.1016/j. compositesa.2011.04.006.

Enviado: Jun. 04, 2014 Reenviado: Ago. 19, 2014 Aceito: Out. 10, 2014 